\title{
Far-UV Fe Emission as Proxy of Eddington Ratios
}

\author{
Wei Zheng $^{1 \star}$ \\ ${ }^{1}$ Department of Physics and Astronomy, Johns Hopkins University, Baltimore, MD 21218, USA
}

17 June 2021

\begin{abstract}
The Eddington ratio is a key parameter that governs the diversity of quasar properties. It can be scaled with a strong anti-correlation between optical Fe $\mathrm{II}_{\mathrm{opt}}$ and [O $\left.\mathrm{III}\right]$ emission. In search of such indicators in the far-UV band, the HST far-UV spectra of 150 low-redshift quasars are analyzed in combination with their optical SDSS counterparts. The strength of $\mathrm{Fe}$ II $+\mathrm{Fe}$ in $\lambda 1123$ emission is significantly correlated with that of Fe $\mathrm{II}_{\mathrm{opt}}$. A moderate correlation may also exist between Fe Iг $\lambda 1071$ and Fe II opt. The finding opens the possibility that far-UV Fe II emission may serve as a new gauge of the Eddington ratios. The high- and low-ionization lines in the far-UV band display different patterns: for the quasars with higher Eddington ratios, the low-ionization UV lines are stronger, and the high-ionization lines are broader and weaker.
\end{abstract}

Key words: galaxies: quasars: emission line - quasars: general

\section{INTRODUCTION}

The presence of emission lines is characteristic in the broadband spectra of active galactic nuclei (AGN). The line profiles and strengths serve as essential diagnostics of the physical conditions in the vicinity of central black holes. It is generally believed that the large dispersion of line widths is mainly attributed to an orientation effect (Jarvis \& McLure 2006; Shen \& Ho 2014), and line widths are often used to classify AGNs (Urry \& Padovani 1995). One of the most significant correlations between line strengths is with the optical Fe II emission: Boroson \& Green (1992) applied the Principal Component Analysis (PCA) to the optical spectra of 87 low-redshift quasars $(z \lesssim 0.5)$ to find a strong anti-correlation between the intensities of Fe II $\lambda 4565$ [Opt 37+38 multiplets (Vanden Berk et al. 2001); Fe II opt hereafter] and [O III] $\lambda 5007$ emission. Boroson (2002) suggested that the underlying reason for this "Eigenvalue 1" is the Eddington ratios for these quasars: the ones with higher Eddington ratios display stronger $\mathrm{Fe}_{\mathrm{II}} \mathrm{opt}$ and narrower $\mathrm{H} \beta$ profiles (Sulentic et al. 2002; Marziani \& Sulentic 2014). These correlations between $\mathrm{Fe} \mathrm{II}_{\mathrm{opt}} / \mathrm{H} \beta$ ratios, $\mathrm{H} \beta$ line widths and Eddington ratios are supported by the work of Shen \& Ho (2014), which is based on a considerably larger database of approximately 20,000 quasars in the Sloan Digital Sky Survey (SDSS, York et al. 2000; Shen et al. 2011).

The majority of quasars are between redshifts $2-3$, and their Fe IIopt emission is shifted into the near-infrared band and beyond the SDSS scope. For these high-redshift quasars, however, their Ly $\alpha$ emission is shifted into the optical band and becomes readily observable. It is, therefore, interesting to search for far-UV (FUV) emission that is correlated to Eigenvalue 1. Several studies have characterized the UV emission lines, exploring the trend of their properties from the optical to UV and the possibility of estimating the masses of central black holes by UV emission lines (McLure \& Jarvis 2002; Kuraszkiewicz et al. 2004; Wang et al. 2009).

The UV spectra of quasars are rich in Fe II and Fe II emission (Wills, Netzer \& Wills 1980; Laor et al. 1997; Vestergaard \& Wilkes 2001; Vanden Berk et al. 2001). While a correlation between Fe II features in the near-UV and optical bands is known (Sameshima et al. 2011; Kovacěvić-Dojčinović \& Popović 2015; Le \& Woo et al. 2019), no such a correlation, to our knowledge, has been established in the FUV band. An interesting and potentially important FUV feature is the pair of Fe II $\lambda 1071$ and Fe II+Fe III $\lambda 1123$ emission: a PCA of Suzuki (2006, S06 hereafter) grouped 50 low-redshift quasars $(z \lesssim 1)$ into four classes with significantly different Ly $\alpha$ emission. Note the difference in this pair of weak Fe II/Fe III emission: in Table 2 of S6, the average equivalent width (EW) of Fe II $\lambda 1071$ is $0.9 \pm 0.9 \AA$ and that for Fe II + Fe III $\lambda 1123$ is $0.1 \pm 0.1 \AA$ for Class-I quasars. As a comparison, these values are $2.7 \pm 1.5$ and $2.2 \pm 1.1 \AA$, respectively, for Class-III quasars. However, this work did not include optical spectra. Other studies with measurements in both far-UV (FUV) and optical bands (in the restframe, and hereafter)

* E-mail: wzheng@jhu.edu 
were based on small sample sizes of $\sim 20$ quasars and with no measurements below the Ly $\alpha$ wavelengths (Shang et al. 2007; Vietril et al. 2020).

A decade after the deployment of the Cosmic Origin Spectrograph (COS, Green et al. 2012), the UV database of quasars has increased significantly. This study utilizes the archival spectra of 150 low-redshift SDSS quasars and compares the properties of FUV and optical emission lines. With a larger and better database, it uses optical Fe in emission to scale its UV counterparts, consolidates the preliminary S06 results on Fe II $\lambda 1071$ and Fe II+Fe III $\lambda 1123$, and explores their potential to serve as a proxy of Eddington ratios.

\section{DATA ANALYSES}

A sample selection of UV and optical spectra was carried out by cross-checking the SDSS quasar catalogs (Schneider et al. 2010; Pâris et al. 2014, 2017, 2018) with the Hubble Space Telescope (HST) server at the Space Telescope Science Institute (STScI). The search within $3^{\prime \prime}$ between the optical and UV coordinates yielded 418 SDSS quasars of $0.05<z<0.8$. The selected redshift range assures a sufficient coverage of the redshifted Fe II opt and [O III] $\lambda 5007$ lines as well as a potential coverage of $\mathrm{O}$ VI $\lambda 1034$, Fe II $\lambda 1071$ and Fe II+Fe III $\lambda 1123$. For simplicity, the Fe II+Fe III $\lambda 1123$ emission is referred to as Feuv hereafter. The archival HST/UV spectra are not as homogeneous as the SDSS, as they were taken with various spectral resolutions and wavelength coverage. The majority of UV spectra (318) were obtained with the COS instrument, and the rest were with three other HST spectrographs (FOS, HRS, and STIS). If a quasar has been observed with COS and other UV spectrographs in a similar wavelength range, its COS data were used. If multiple observations with the same spectrograph were made, the spectra were combined with the weights of exposure times. The data were then rebinned with a pixel size of $0.5 \AA$ in the observer's frame. Some data were rejected because of their low signal-to-noise ratios (average $S / N<5$ per pixel, as calculated from the rebinned data arrays of fluxes and errors in the fitting windows between 995 and $1170 \AA$ ), significantly incomplete (i.e., missing more than half of the emission profile) O vi $\lambda 1034$ or Fe $\mathrm{UV}_{\mathrm{UV}}$ profiles, or seriously contaminating broad absorption lines. The final sample consists of 150 quasars between $0.1<z<0.7$, of which 132 are with COS spectra. As a comparison, the FOS sample of Kovacěvić et al. (2010) consists of 61 quasars in the same redshift range, and that of S06 is 31. The optical spectra were retrieved from the SDSS DR14 server. All data consist of a pair of flux and error arrays. The average redshift is 0.321 , and the mean redshift 0.270 .

Spectral analyses of continua and emission lines were carried out using the task Specfit (Kriss 1994). Absorption features of EW $>0.1 \AA$ were marked and excluded in the fitting wavelength windows. For each optical spectrum, the following components were first applied: a power-law continuum, dual broad Gaussian components for H $\beta$, a Gaussian component for He in $\lambda 4686$. A set of Fe II opt templates of Véron-Cetty et al. (2004), convolved with Lorentzian profiles between 1000 and $8000 \mathrm{~km} \mathrm{~s}^{-1}$, were generated and then divided into two sets by wavelengths: one to fit Fe II opt at wavelengths $<4700 \AA$, and another companion set of Fe II templates at wavelengths $>4800 \AA$ to fit the Fe II multiplets at $\sim 4900-5050 \AA$ (Opt 42; Vanden Berk et al. 2001). Specfit took these two sets of user-supplied components of velocity-broadened templates along with two free parameters: the scaling factor in fluxes and redshift for wavelengths. The scaling factors were allowed to vary independently, and the redshifts for these Fe II templates were set as the systemic redshifts and allowed to vary slightly within the range of $|d z|<0.005(1+z)$. The best fitting results included the templates' broadening velocities, scaling factors and redshifts.

For Gaussian components, their centroid wavelengths, fluxes, and widths were set free, except for the [O III] $\lambda \lambda 4959 / 5007$ doublets: their ratios of fluxes and centroid wavelengths were tied to the intrinsic atomic data and their line widths were identical. Note that all the centroid wavelengths in Specfit fitting were redshifted, but their values shown in this paper are in the restframe. The broad $\mathrm{H} \beta$ components (also for other pairs of dual components) were of Full Width at Half Maximum (FWHM, or "width") $\gtrsim 3000 \mathrm{~km} \mathrm{~s}^{-1}$, and $1500-3000 \mathrm{~km} \mathrm{~s}^{-1}$. If a spectrum displays a discrete narrow $\mathrm{H} \beta$ feature and the fit narrow $\mathrm{H} \beta$ component showed an FWHM $\lesssim 1500 \mathrm{~km} \mathrm{~s}^{-1}$, an additional narrow $\mathrm{H} \beta$ component of $\mathrm{FWHM}<1500 \mathrm{kms} \mathrm{s}^{-1}$ was added. Narrow components of FWHM $<1500 \mathrm{~km} \mathrm{~s}^{-1}$ (Zakamska et al. 2003) were excluded in calculating broad H $\beta$ fluxes as they are unlikely formed in broad-line regions.

Fe II emission is weak and broad, and its measurements depend heavily on the accuracy of an underlying continuum. Unfortunately, there is no emission-free region in the vicinity of $\mathrm{H} \beta$. Two narrow windows at $4180-4220$ and $5080-5120 \AA$ were chosen to provide a baseline of the underlying continuum. Overall, the optical fitting windows are $4180-4220$ and $4415-5120 \AA$, excluding $\mathrm{H} \gamma$. In the FUV band, the nominal fitting range was between 995 and $1170 \AA$ if data were available. The continuum windows free of emission lines were $995-1005,1090-1100$, and $1140-1150 \AA$, and at least two of them should be available for fitting. If the data extend to $1280 \AA$, an additional window of $1270-1280 \AA$ was added to improve the accuracy. Ly $\alpha$ emission was fitted with dual Gaussian components along with dual N v $\lambda 1240$ components. The centroid wavelengths of $\mathrm{N}$ v $\lambda 1240$ components were tied to their respective Ly $\alpha$ counterparts by the intrinsic atomic values. Additional complications arise from very broad red wings of $\mathrm{O}$ vi $\lambda 1034$ that may extend beyond Fe II $\lambda 1071$. The Ly $\beta$ component was a Gaussian with a centroid at $\sim 1026 \AA$, and O vi $\lambda 1034$ with dual broad components: one at FWHM $1500-3000 \mathrm{kms}^{-1}$, and another at $>3000 \mathrm{~km} \mathrm{~s}^{-1}$. The wavelength ratio of Ly $\beta$ and the broader O VI $\lambda 1034$ component was fixed based on their 
intrinsic atomic data, and the Ly $\beta$ width was tied to that of the broader Ly $\alpha$ component. The two FUV Fe in features were each fit with a Gaussian of FWHM $\lesssim 7000 \mathrm{~km} \mathrm{~s}^{-1}$. Figure 1 illustrates an example of the optical and FUV spectra of a Fe II-strong quasar with the fitting components, residues and fitting windows. Figure 2 displays the spectra of ten quasars with the highest $\mathrm{Fe} \mathrm{II}_{\mathrm{opt}} / \mathrm{H} \beta$ or $\mathrm{Fe}_{\mathrm{UV}} /(\mathrm{Ly} \beta+\mathrm{O} \mathrm{VI})$ ratios, and Figure 3 for ten other quasars with the lowest Fe $\mathrm{II}_{\mathrm{opt}} / \mathrm{H} \beta$ ratios.

To calculate the FWHM and its error from a summed profile of multiple Gaussian components $(\mathrm{H} \beta$, Ly $\alpha$ and Ly $\beta+\mathrm{O}$ vi) the widths of every component were generated randomly 200 times from its FWHM and error, and then 200 summed profiles were made. For each summed profile, its peak position was determined, and then the FWHM was measured at the half-peak positions. This procedure was repeated 200 times for each line, and the average and standard deviation of these measurements were tabulated as the FWHM and its error.

The emission-line measurements in these selected quasars are listed in Tables 1 and 3, respectively. The last column in these tables shows whether a quasar has been in the S06 sample. The errors in these tables were calculated from the Specfit results, and those of line ratios propagated through mathematical formulas for the merging components. To calculate the propagated errors for ratios between two variables, their relative errors were in an RMS relation. Namely, if $\mathrm{A}, \Delta A$ and $\mathrm{B}, \Delta B$ are the two variables with their respective errors, the errors of ratio $\mathrm{C}=(\mathrm{A} / \mathrm{B})$ is interpreted as $\Delta C / C=\sqrt{(\Delta A / A)^{2}+(\Delta B / B)^{2}}$. For $\mathrm{Ly} \beta+\mathrm{O}$ vi, one Ly $\beta$ and two $\mathrm{O}$ vi components were included, and, for $\mathrm{H} \beta, \mathrm{O}$ vi and Ly $\alpha$, two components were included. Note that the actual errors are likely larger than the tabulated values, as Specfit values may not include all possible uncertainties, especially when strong absorption is present near a line center. In Tables 2 and 4, the line widths of $\mathrm{O}$ vi+Ly $\beta$, Ly $\alpha$, and $\mathrm{H} \beta$ in the two groups are tabulated, respectively.

\section{DISCUSSION}

\subsection{Relation between UV and optical Fe iI ratios}

The Fe Uv emission is marked as Fe III (UV 1) by Laor et al. (1997); Vanden Berk et al. (2001); Harris et al. (2016), and as Feuv by S06. Figure 4 displays the Feuv/(Ly $\beta+\mathrm{O}$ vi) ratios vs. the Fe II opt $/ \mathrm{H} \beta$ ratios for the sample. The Pearson coefficient of $R=0.748$ suggests a significant correlation, with a probability ( $\mathrm{P}$-value) of $<10^{-10}$ for an uncorrelated dataset producing such a distribution. The linear regression slope is 0.307 and an intercept is -0.068 . To understand the effect of data dispersion to the correlation, the Pearson test was repeated 100 times with simulated data errors. In each run, the data points were modified with errors, which were generated randomly under a normal distribution for the given standard deviations. The Pearson coefficients were between 0.58 and 0.75 , with an average value of $\bar{R}=0.68 \pm 0.04$. Even at the lowest value of $R=0.58$, the P-value is still smaller than $10^{-10}$.

The trend suggests that quasars with higher Feuv emission likely show high Fe II $\mathrm{I}_{\mathrm{opt}}$, thus providing an epoxy of the Eddington ratios in high-redshift quasars where optical emission is not readily observable. Note that the dispersion shown in Figure 4 is significant; therefore, a prediction of the Eddington ratio for a given quasar would still be uncertain. At Fe IIopt $/ \mathrm{H} \beta$ ratios $\lesssim 0.7$, the Feuv features in many quasars are not detected. Some of these quasars with very low $\mathrm{Fe} \mathrm{II}_{\mathrm{opt}} / \mathrm{H} \beta$ ratios may be referred as "Pop B quasars" (Marziani et al. 2018). If the data points at Feuv/(Ly $\beta+\mathrm{O}$ vi) $<0.025$ are excluded as their values are below the average errors in this section of data, the remaining data of 90 quasars yield the Pearson coefficient of 0.706 and the $\mathrm{P}$-value at $<10^{-10}$. For a group of quasars with low Feuv/(Ly $\beta+\mathrm{O}$ vi) ratios $(<0.1)$, their average Fe II opt $/ \mathrm{H} \beta$ ratio is $0.39 \pm 0.21$. Comparing with Figure 2 of Marziani et al. (2018), these quasars are of type B1 and A1. For the group with high $\mathrm{Feuv}_{\mathrm{u}} /(\mathrm{Ly} \beta+\mathrm{O} \mathrm{vI})$ ratios $(>0.3)$, their $\mathrm{Fe} \mathrm{II}_{\mathrm{opt}} / \mathrm{H} \beta$ ratio is $1.06 \pm 0.21$. These quasars are mostly of type A3. The Eddington ratios of these two groups, as judged from the optical Fe II strengths, are noticeably different.

Figure 4 is plotted in line ratios, following the convention in most publications on Fe II properties. Since both $H S T$ and SDSS spectra are photometric (Hirschauer et al. 2021; Ivezić et al. 2004), it would be interesting to directly compare the FUV and optical Fe II fluxes, as shown in Figure 5. While the data display significant dispersion, the Pearson coefficient between the $\mathrm{Fe}_{\mathrm{UV}}$ and $\mathrm{Fe} \mathrm{II}_{\mathrm{opt}}$ fluxes is 0.763 , and their linear relationcan be described as $\mathrm{Fe}_{\mathrm{UV}}=0.381$ Fe $\mathrm{II}_{\mathrm{opt}}-1.9 \quad$ where fluxes are in units of $10^{-15} \mathrm{ergs} \mathrm{s}^{-1} \mathrm{~cm}^{-2}$. The corresponding P-value is smaller than $10^{-10}$. The tests with simulated data errors yielded an average value of $\bar{R}=0.73 \pm 0.02$, corresponding to P-values smaller than $10^{-10}$. The trend in this figure supports that the strengths of Feuv and Fe II $\mathrm{opt}_{\mathrm{Ut}}$ are indeed correlated.

The emission feature around $1074 \AA$ was identified as Ar IV (Vanden Berk et al. 2001), as "unknown" by Harris et al. (2016), and as Fe II $\lambda 1071$ by S06. Between Fe II $\lambda 1071 /(\mathrm{Ly} \beta+\mathrm{O}$ vi) and Fe II opt $/ \mathrm{H} \beta$, the Pearson coefficient is 0.395 , and the P-value is $<6 \times 10^{-7}$. The regression line slope is 0.164 and the intercept 0.11 . Since there are no other Ar lines and since the strength of this feature displays a moderate correlation with Fe $\mathrm{II}_{\mathrm{opt}}$, it is likely a Fe II feature. The lower significance for Fe II $\lambda 1071$ is probably attributed to contaminations from the red broad $\mathrm{O}$ vi $\lambda 1034$ wing (Figure 6).

MNRAS 000, 1-17 (2021) 


\subsection{Properties of other FUV lines}

The spectra of Fe II-strong quasars in Table 1 and Figure 2 were combined after normalizing with the fluxes around $1100 \AA$. Similarly, a combined spectrum was made for ten Fe II-weak quasars in Table 3 and Figure 3. As shown in Figure 7 , the Ly $\alpha$ emission in Fe II-weak quasars displays a stronger narrow component. A Specfit fit with dual components for Ly $\alpha$ and $\mathrm{O} v \mathrm{v}+\mathrm{Ly} \beta$ and single components for other emission lines was carried out. The EWs of narrow Ly $\alpha$ components are $11.6 \pm 1.3$ $\AA$ for Fe II-strong quasars and $34.0 \pm 2.5 \AA$ for Fe II-weak quasars. The respective FeUv EWs are $4.5 \pm 0.7$ and $0.3 \pm 1.0 \AA$. To check if the trend is a small-sample fluctuation, the same test was carried out for 20 Fe II-strong quasars and other 20 Fe II-weak ones. The EW of narrow Ly $\alpha$ components are $18.1 \pm 1.3$ and $33.0 \pm 1.8 \AA$, respectively. Since there is a minimal overlap between these quasars and those in S06, this trend provides additional support to the results in S06. It suggests that Class-I quasars are Fe II weak, Ly $\alpha$ strong and have low Eddington ratios, in contrast to their Class-III counterparts.

Sulentic et al. (2002) studied the spectral features for different groups in the diagram of Fe II opt $/ \mathrm{H} \beta$ ratios vs. H $\beta$ FWHM. These groups display distinct $\mathrm{Fe} \mathrm{II}_{\mathrm{opt}}$ strengths as well as $\mathrm{H} \beta$ widths. Based on the correlation between Fe $\mathrm{UV}_{\mathrm{UV}}$ and $\mathrm{Fe} \mathrm{II}_{\mathrm{Opt}}$ (Figures 4 and 5), it may be possible to link the UV classes of S06 with the optical groups of Sulentic et al. (2002): Class-I quasars show absent $\mathrm{Fe}_{\mathrm{UV}}$ and are hence related to the B-type in the optical. Class-III quasars show significant Fe $\mathrm{UV}_{\mathrm{V}}$ and are related to the A-3 type.

It is also noted in Figure 7 that the $\mathrm{C}$ II $\lambda 1335$ emission is correlated with Fe II strengths. Between the ratios of $\mathrm{C}$ II $\lambda 1335 / \mathrm{Ly} \alpha$ and $\mathrm{Fe} \mathrm{II}_{\mathrm{opt}} / \mathrm{H} \beta$ ratios of 51 objects, the Pearson coefficient of 0.475 and the P-value of $0.04 \%$ suggest a moderate correlation.

Note that both the FeUv and C II $\lambda 1335$ emission is of low ionization, and their strengths are different from major FUV emission lines, which are of high ionization. Emission lines of different ionization levels are believed to originate in separated regions (Shang et al. 2007; Richards et al. 2011; Braibant et al. 2016). The results in this study suggest that some low-ionization lines may scale Eddington ratios.

$\mathrm{Mg}$ II $\lambda 2798$ emission is of low ionization; therefore, it is conceivable that the FWHMs of Mg II $\lambda 2798$ are similar to those of $\mathrm{H} \beta$ (McLure \& Jarvis 2002; Wang et al. 2009), and they were used to estimate the central black-hole masses. Śniegowska et al. (2020) studied near-UV (NUV) lines and compared them with Fe IIopt/H $\beta$. They found that the Mg II $\lambda 2798$ properties bear resemblance with $\mathrm{H} \beta$, and the NUV Fe in strengths are correlated to Fe II opt. However, major FUV lines are of high ionization. Attempts have been made to explore the possibility of using FUV emission-line widths to scale the accretion rate, and the results remain unconvincing (Shemmer et al. 2004; Martínez-Aldama, et al. 2017; Sulentic et al. 2017), as the C IV $\lambda 1549$ widths are significantly different from those of $\mathrm{H} \beta$. As shown in Figure 8, the FWHM of Ly $\alpha$ and $\mathrm{H} \beta$ are uncorrelated, with the Pearson coefficient of 0.079 and the P-value of $46 \%$ (90 data points).

The Fe II-strong quasars, as shown by red symbols, have narrow $\mathrm{H} \beta$ widths and randomly distributed Ly $\alpha$ widths. The Fe IIweak quasars, as shown in green symbols, have narrow Ly $\alpha$ widths and moderate-to-broad H $\beta$ widths. Given the significant dispersion, the central masses and accretion rates derived from Ly $\alpha$ would be inconsistent with those from $\mathrm{H} \beta$.

It has been suggested (Shen \& Ho 2014; Marziani et al. 2018) that strong Fe $\mathrm{II}_{\mathrm{opt}} / \mathrm{H} \beta$ ratios are related to high accretion rates. It is also known that strong $\mathrm{Fe} \mathrm{II}_{\mathrm{opt}}$ is associated with narrow $\mathrm{H} \beta$ (Sulentic et al. 2002). The nature of a broad span of $\mathrm{H} \beta$ line widths is complex: while they are attributed to an orientation effect, other effects may also be at work. In virialized cases, the $\mathrm{H} \beta$ line widths are also related to the black-hole masses and accretion rates (Sulentic et al. 2017). In other words, there appear to be intrinsic difference between Type-A and B quasars in Figure 2 of Marziani et al. (2018). In Figure 9, the widths of $\mathrm{O} v \mathrm{v}+\mathrm{Ly} \beta$ are plotted vs. the ratios of $\mathrm{Fe} \mathrm{II}_{\mathrm{opt}} / \mathrm{H} \beta$. Strong Feuv seems to be associated with broader $\mathrm{O}$ vi $+\mathrm{Ly} \beta$. This trend illustrates the difficulty of using the widths of Ly $\alpha$ and other FUV lines (Richards et al. 2011) to estimate the accretion rates. For this sample, the Pearson efficient between the widths of $\mathrm{O}$ vi $+\mathrm{Ly} \beta$ and $\mathrm{H} \beta$ is merely 0.065 (P-value $43 \%$ ).

\subsection{Redshift effect}

Between redshifts $0.1-0.7$, the cosmic distances span a large range by a factor of $\sim 5$, and this quasar sample covers the absolute luminosities between -21.3 and -26.4 . To check if the observed trend is redshift dependent, a statistical test was carried out between the $\mathrm{Fe} \mathrm{II}_{\mathrm{opt}} / \mathrm{H} \beta$ ratios and redshifts. It yielded the Pearson coefficient of 0.064 and the $\mathrm{P}$-value of $43 \%$, suggesting a null correlation.

The correlation between Fe $\mathrm{II}_{\mathrm{opt}} / \mathrm{H} \beta$ and the absolute magnitude is also null, with a Pearson coefficient of -0.023 and the P-value of $77 \%$. Note that the average redshift in the Fe II-weak group (Table 3) is slightly higher than that in the Fe II-strong group (Table 1). Overall, the correlation shown in Figures 4 and 5 is not attributed to redshifts or luminosities.

\section{SUMMARY}

Based on a large database of FUV and optical spectra of low-redshift quasars, we find that (1) the relation between FUV and optical emission lines is complex: the line widths of Ly $\alpha$ are uncorrelated with those of $\mathrm{H} \beta$, making it difficult to use 
Ly $\alpha$ to estimate the masses of central black holes; (2) the equivalent widths of high- and low-ionization lines exhibit opposite correlations with $\mathrm{Fe} \mathrm{II}_{\mathrm{opt}}$; (3) the significant correlation between $\mathrm{Fe}_{\mathrm{UV}}$ and $\mathrm{Fe}_{\mathrm{II}}$ opt strengths makes it possible to use the FUV Fe II emission as a proxy of the Eddington ratios; and (4) the quasars with high Eddington ratios tend to display broader Ly $\alpha$ and $\mathrm{O}$ vi emission, contrary to a trend in the optical band.

A proxy of the Eddington ratios would be valuable at higher redshifts, when Fe $\mathrm{II}_{\mathrm{opt}}$ and $\mathrm{H} \beta$ emission features are redshifted out of the optical band. The results suggest that the quasar classification of S06 in the FUV band is driven by the Eddington ratios, consistent with previous results of the optical and X-ray bands.

\section{ACKNOWLEDGMENTS}

The author thanks the anonymous referee for many thoughtful comments.

Funding for the SDSS and SDSS-II has been provided by the Alfred P. Sloan Foundation, the Participating Institutions, the National Science Foundation, the U.S. Department of Energy, the National Aeronautics and Space Administration, the Japanese Monbukagakusho, the Max Planck Society, and the Higher Education Funding Council for England. The SDSS Web Site is http://www.sdss.org/.

This research has made use of the NASA/IPAC Extragalactic Database (NED), which is operated by the Jet Propulsion Laboratory, California Institute of Technology, under contract with the National Aeronautics and Space Administration (NASA). It is based on observations made with the NASA/ESA Hubble Space Telescope, obtained from the data archive at the Space Telescope Science Institute. STScI is operated by the Association of Universities for Research in Astronomy, Inc. under NASA contract NAS 5-26555. Some of the data presented in this paper were obtained from the Mikulski Archive for Space Telescope (MAST). STScI is operated by the Association of Universities for Research in Astronomy, Inc., under NASA contract NAS5-26555.

\section{DATA AVAILABILITY STATEMENT}

All the data and software in this report are public.

\section{REFERENCES}

Boroson, T. A., 2002, ApJ, 565, 78

Boroson, T. A. \& Green, R. F., 1992, ApJS, 80, 109

Braibant, L., Hutsemékers, Sluse, D., Anguita, T., A\&A, 2016, 592, A23

Green, J. C., Froning, C. S., Osterman, S. et al., 2012, ApJ, 744, 60

Harris, D. W., Jesen, T. W., Suzuki, N., et al., 2016, AJ, 151, 155

Hirschauer, A. S. et al., 2021, Cosmic Origins Spectrograph Instrument Handbook, Version 13.0 (Baltimore: STScI)

Ivezić, Ž., Lupton, R., Boroski, B. et al., 2004, Astron. Nachr., 325, 583

Jarvis, M. J. \& McLure, R. J., 2006, MNRAS, 369, 182

Kovacěvić, J., Popović, L., Č, Dimitrijević, M. S., 2010, ApJS, 189, 15

Kovacěvić-Dojčinović, J. \& Popović, L., Č, 2015, ApJS, 221, 35

Kriss, G. A., 1994, in Astronomical Data Analysis Software and Systems III, eds. D. R. Crabtree, R. J. Hanisch, \& J. Barnes, (A. S. P. Conf. Series 61, ASP, San Francisco), 437

Kuraszkiewicz, J. K., Green, P. J., Crenshaw, D. M. et al., 2004, ApJS, 150165

Laor, A., Jannuzi, B. T., Green, R. F., Boroson, T. A., 1997, ApJ, 489, 656

Le, H. A. N. \& Woo, J.-H., 2019, ApJ, 887, 236

Martínez-Aldama, M. L., del Olma, A., Marziani, P., et al., 2017, Fr. ASS, 4, 65

Marziani, P., Dultzin, D., Sulentic, J. W., et al., 2018, Front. Astron. Space Sci., 5, 6

Marziani, P. \& Sulentic, J. W., 2014, MNRAS, 442, 1211

McLure, R. J. \& Jarvis, M. J. 2002, MNRAS, 337, 109

Pâris, I., Petitjean, P., Aubourg, É, et al., 2014, A\&A, 563, 54

Pâris, I., Petitjean, P., Aubourg, É, et al., 2018, A\&A, 613, A51

Pâris, I., Petitjean, P., Ross, N., et al., 2017, A\&A, 597, A79

Richards, G. T., Kruczek, N. E., Gallagher, S. C., et al., 2011, AJ, 141, 167

Sameshima, H., Kawara, K., Matsuoka, Y. et al., 2011, MNRAS, 410, 1018

Schneider, D. P., Richards, G. T., Hall, P. B., et al., 2010, AJ, 139, 2360

Shang, Z., Wills, B. J., Wills, D., Brotherton, M. S., 2007, AJ, 134, 294

Shemmer, O., Netzer, H., Maiolino, R. et al., 2004, ApJ, 614, 547

Shen, Y. \& Ho, L. C., 2014, Nature, 513, 210

Shen, Y., Richards, G. T., Strauss, M. A. et al., 2011, ApJS, 194, 45

Śniegowska, M., Kozlowski, S., Czerny, B., et al., 2020, ApJ, 900, 64

Sulentic, J. W., Marziani, P., Zamanov, R., et al., 2002, ApJ, 566, L71 
Sulentic, J. W., del Olmo, A., Marziani, P., et al., 2017, A\&A, 608, A122 Suzuzi, N., 2006, ApJS, 163, 110 (S06)

Urry, C. M., \& Padovani, P., 1995, PASP, 107, 803

Vanden Berk, D. E., Richards, G. T., Bauer, A. et al., 2001, AJ, 122, 549

Véron-Cetty, M.-P., Joly, M. \& Véron, P, M., 2004, A\&A, 417, 515

Vestergaard, M., \& Wilkes, B., 2001, ApJS, 134, 1

Vietri1, G., Mainieri, V., Kakkad, D. et al., 2020, A\&A, 644, A175

Wang, J.-G., Dong, X.-B., Wang, T.-G. et al., 2009, ApJ, 707, 1334

Wills, B. J., Netzer, H., Wills, D., 1980, ApJ, 242, L1

York, D. G., Adelman, J., Anderson, J. E. Jr., et al., 2000, AJ, 120, 1579

Zakamska, N. L., Strauss, M. A., Krolik, J. H., et al., 2003, AJ, 126, 2125 


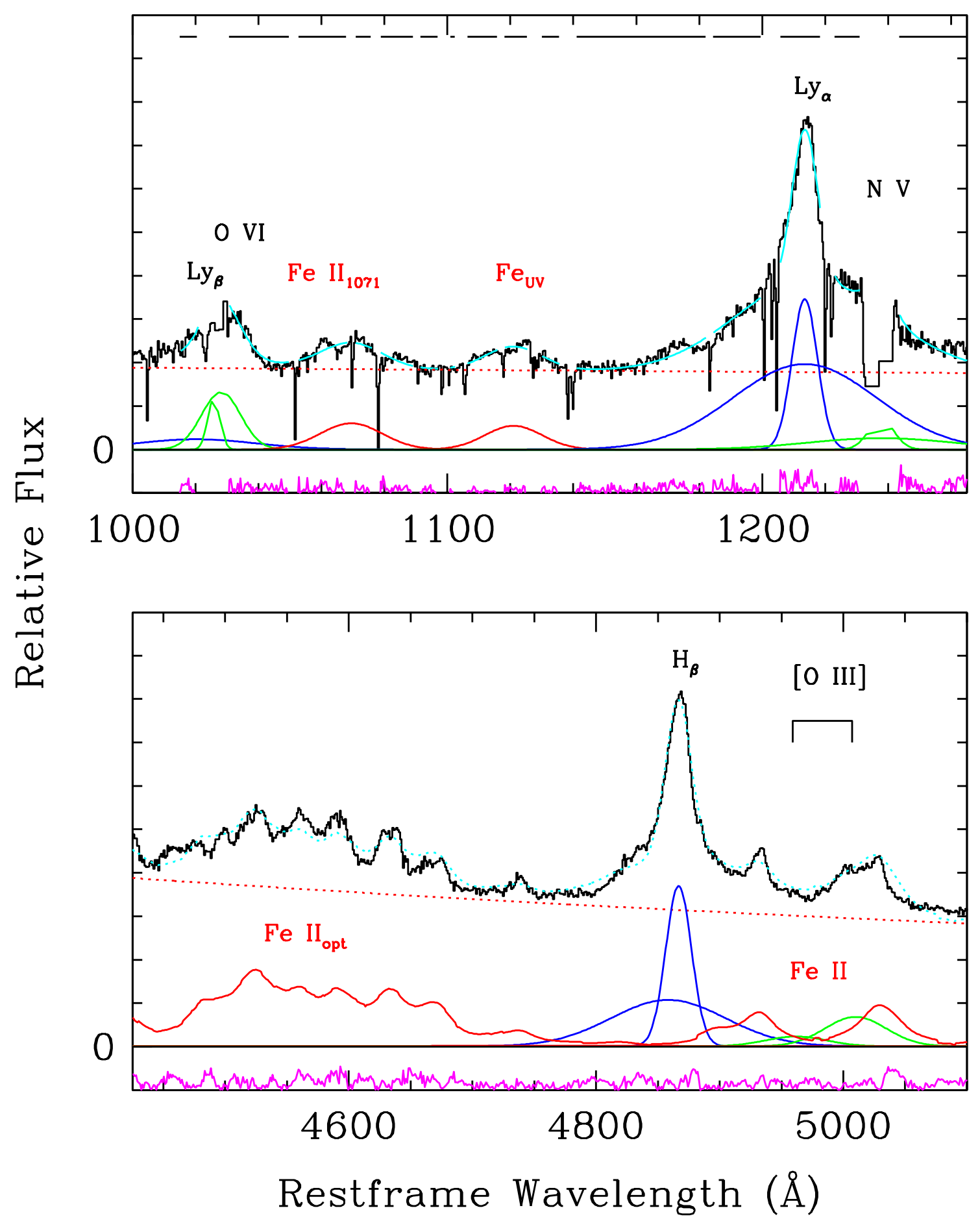

Figure 1. Spectral fit to UV and optical Fe II features in quasar SDSS1619+2543 ( $z=0.2685)$. The UV fitting windows are marked by horizontal bars in the upper panel. The Specfit fitting results are plotted in cyan, the power-law continua in red, and the absolute values of residues in the fitting windows in magenta (with a down shift to avoid overlapping). The Fe II components are plotted in red, hydrogen lines in blue, and other metal (oxygen and nitrogen) lines in green. The optical Fe II features are fit with velocity-convolved templates of Véron-Cetty et al. (2004), with a normalization break at $\sim 4700-4800 \AA$, and the others with Gaussians. The data discontinuity near $1025 \AA$ is the result of geocoronal contamination, and that near $1237 \AA$ an instrumental gap. Note that the narrower components of O VI and $\mathrm{N} \mathrm{V}$ are largely outside the fitting windows; therefore their fitting results are uncertain. 


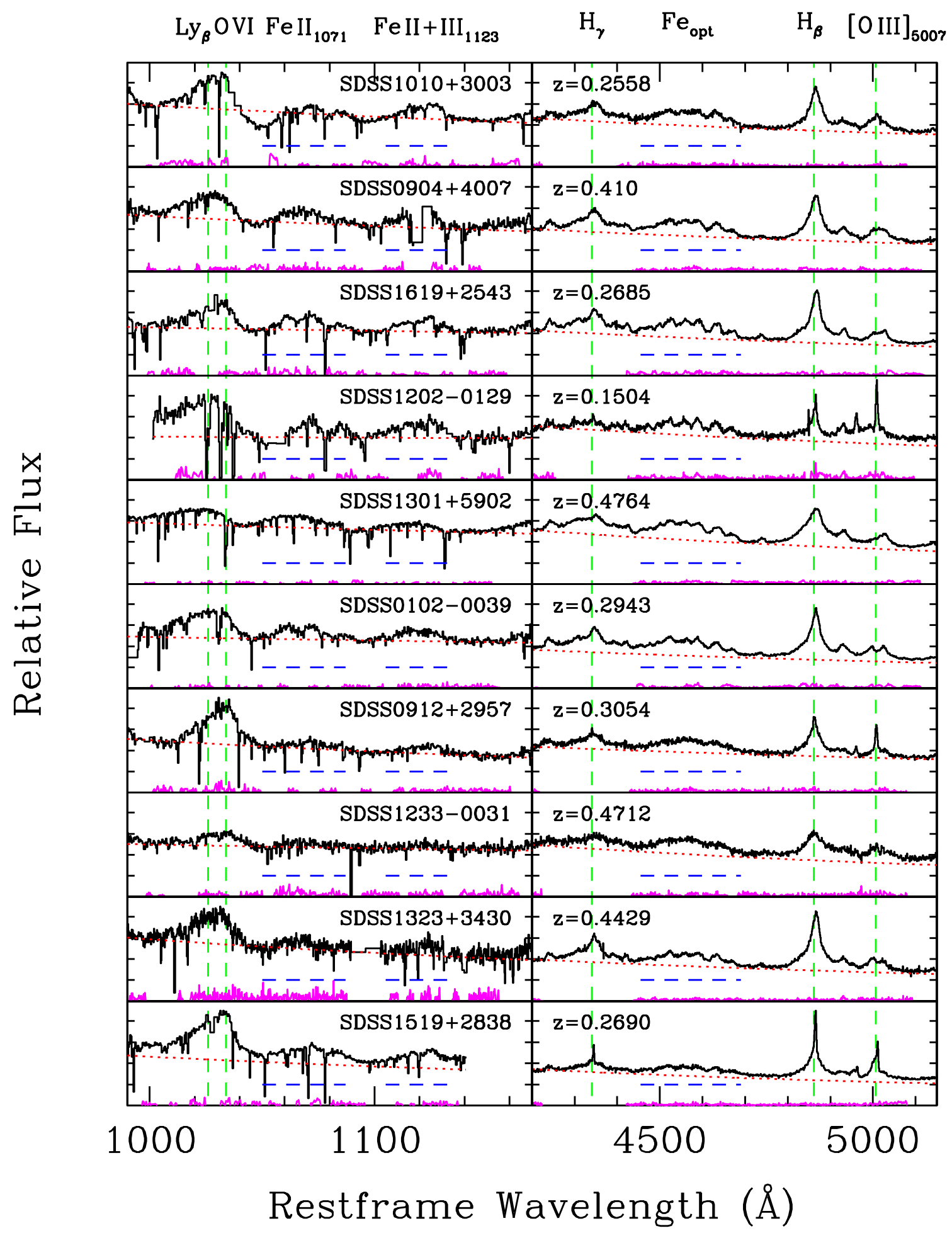

Figure 2. Spectra of ten SDSS quasars with the highest Fe II opt $/ \mathrm{H} \beta$ ratios $(>1.1)$ or FeUv/(Ly $\beta+\mathrm{O}$ VI) ratios $(>0.4 ;$ see Table 1$)$. Fluxes are scaled for proper display. Disconnected data points are instrumental gaps or the results of removing geocoronal contaminations. $\mathrm{Ly} \beta, \mathrm{O}$ vi $\lambda 1034, \mathrm{H} \gamma, \mathrm{H} \beta$, and [O III] $\lambda 5007$ are labeled at the top, and their central wavelengths are marked with vertical green lines. The broad FeUV and Fe II opt features are labeled, and their wavelength ranges are marked with horizontal blue lines. The fitted power-law continua are plotted in red dotted lines, and the absolute values of Specfit residues in the fitting windows are plotted in magenta. 


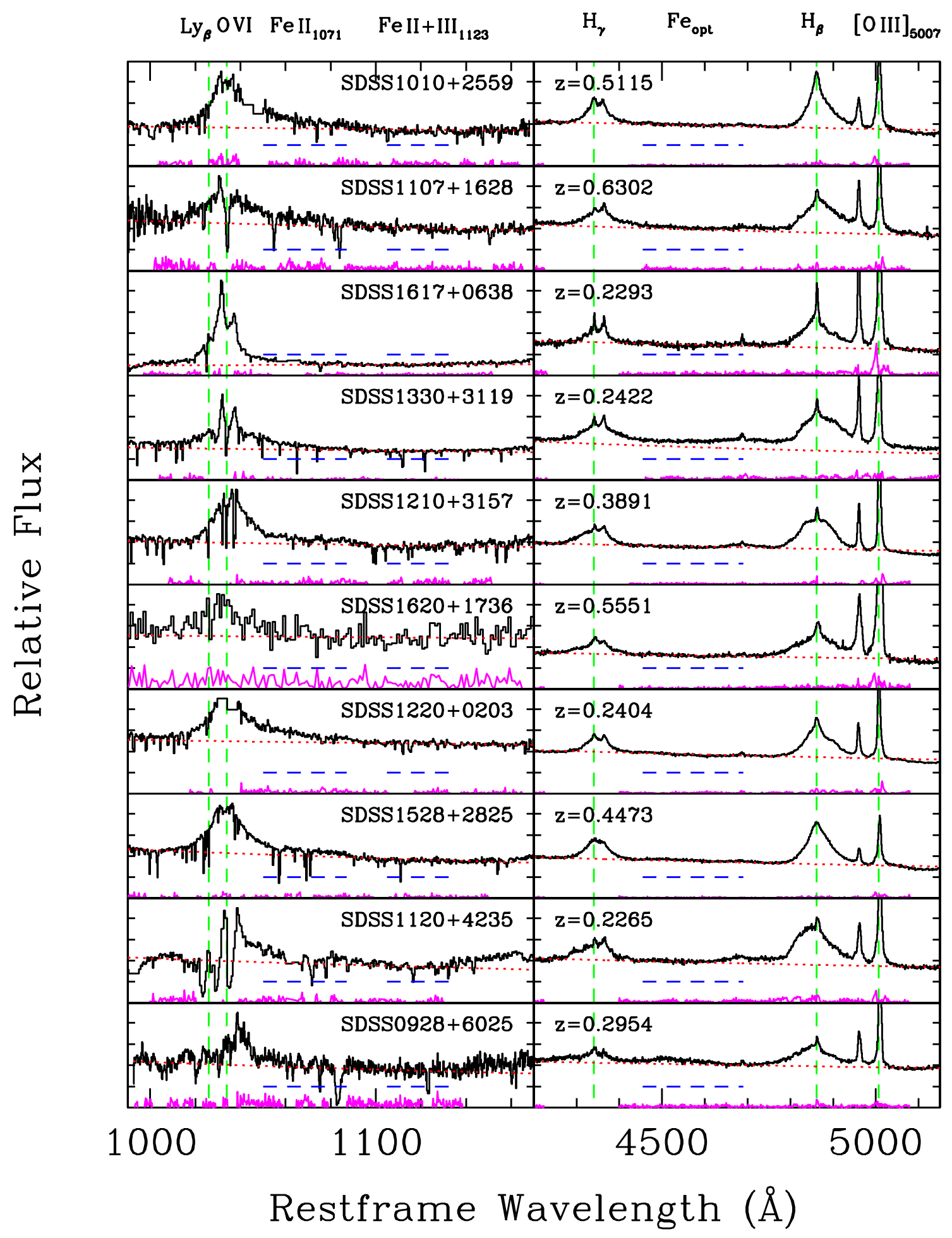

Figure 3. Spectra of ten SDSS quasars with the lowest Fe $\mathrm{II}_{\mathrm{opt}} / \mathrm{H} \beta$ ratios $\leq 0.11$. see Table 3). The same caption as Figure 2 . 


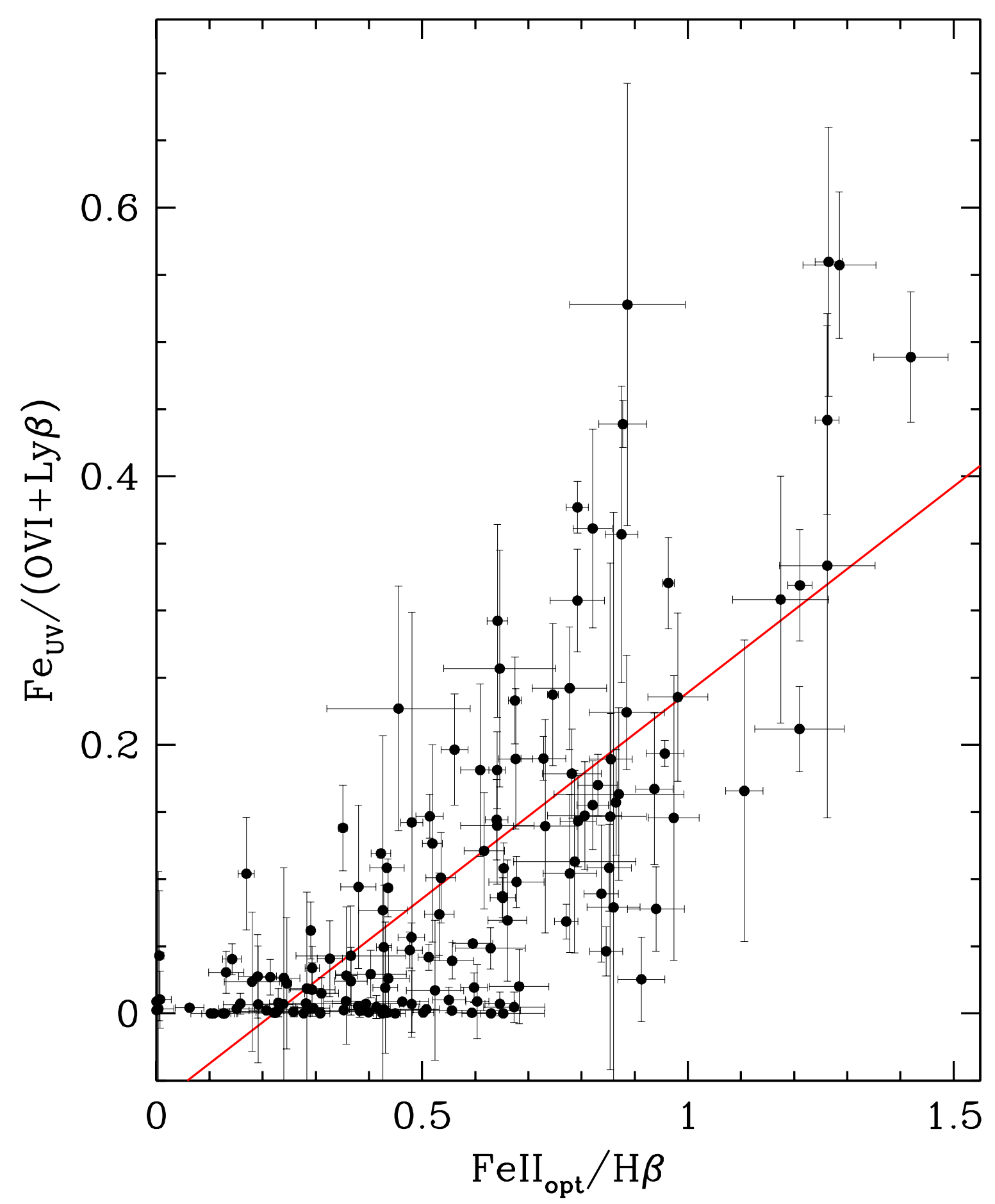

Figure 4. EW ratios of FeUv/(Ly $\beta+\mathrm{O}$ vi $)$ vs. Fe $\mathrm{II}_{\mathrm{opt}} / \mathrm{H} \beta$. The Pearson coefficient of 0.75 suggests a significant correlation between them. The line of best linear regression of slope 0.31 is plotted in red. The errors are propagated from the Specfit fitting results of individual Gaussian components. 


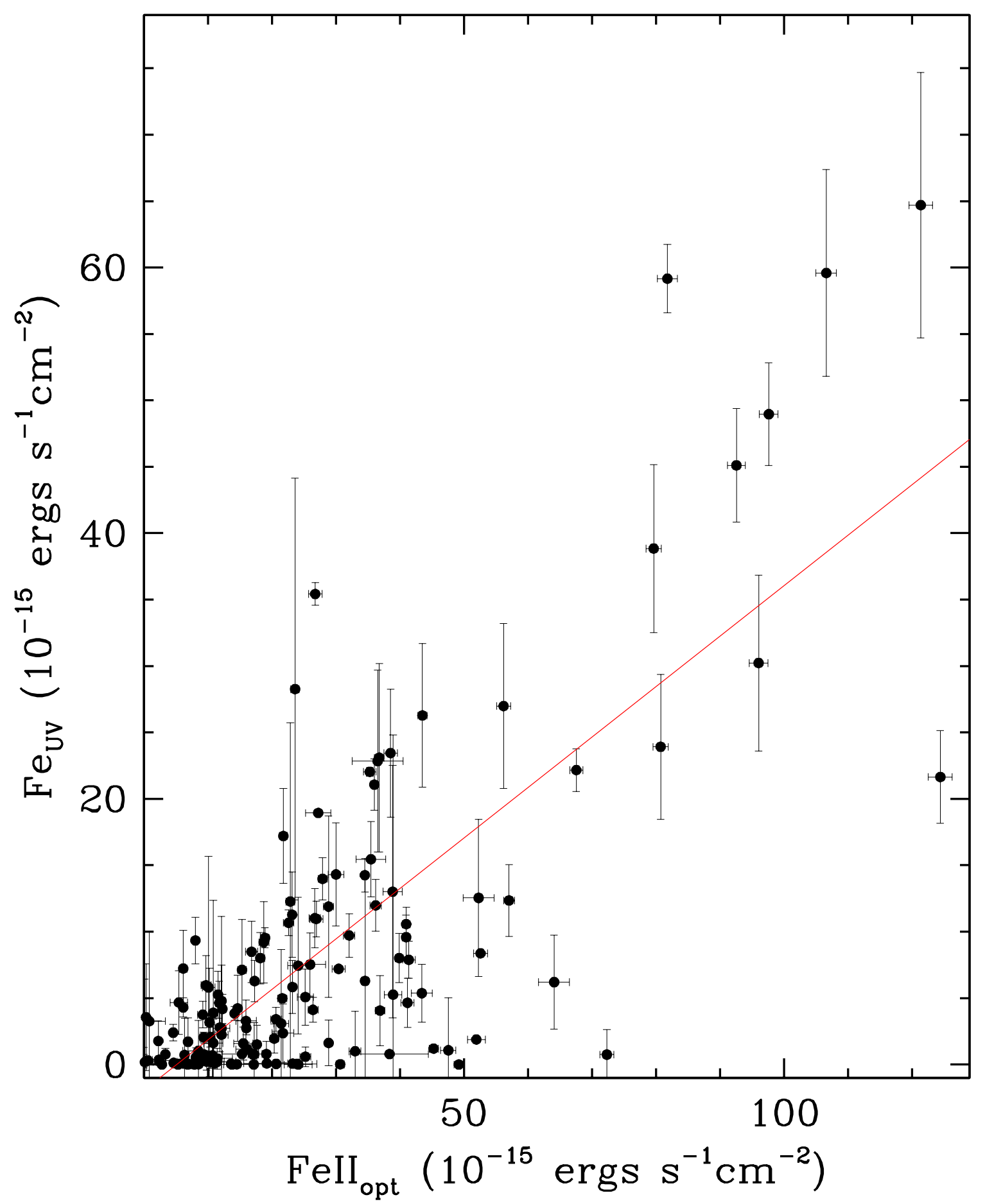

Figure 5. Restframe fluxes of FeUv vs. Fe II $\mathrm{I}_{\mathrm{Upt}}$ emission. The Pearson coefficient of 0.76 suggests a significant correlation. The line of best linear regression of slope 0.38 is plotted in red. 


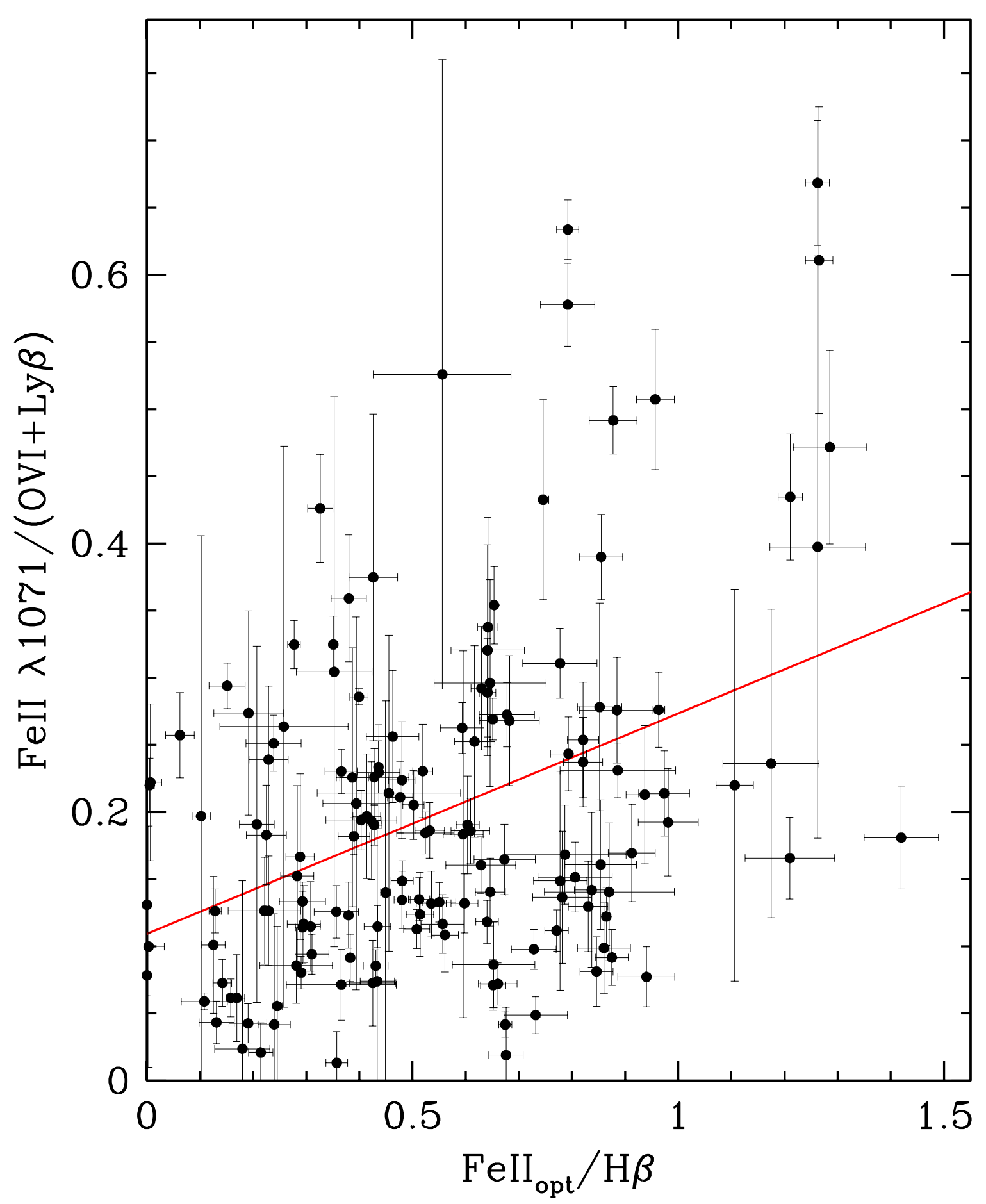

Figure 6. EW ratios of Fe II $\lambda 1071 /(\mathrm{Ly} \beta+\mathrm{O}$ VI) vs. Fe II opt $/ \mathrm{H} \beta$. The Pearson coefficient of 0.40 suggests a moderate correlation between them. The best linear regression of slope of 0.17 is plotted in red. See caption of Figure 4. 


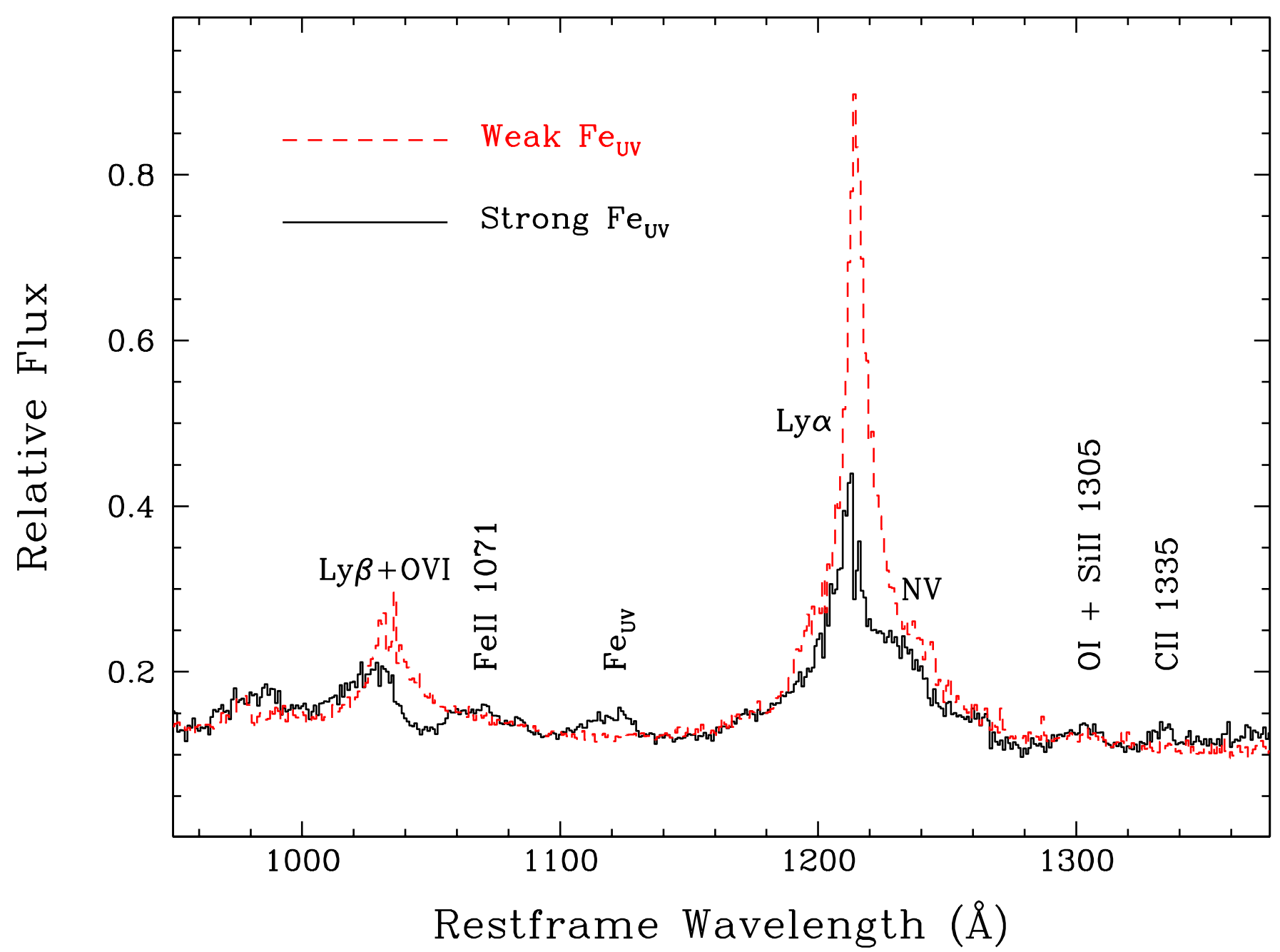

Figure 7. Summed spectra of quasars with different Fe $\mathrm{UV}_{\mathrm{UV}}$ strengths. The spectrum in black is that of the ten strongest Fe II (Figure 2 and Table 1), and that in red dashes of the ten weakest Fe II (Figure 3 and Table 3). Note the difference in C II $\lambda 1335$. 


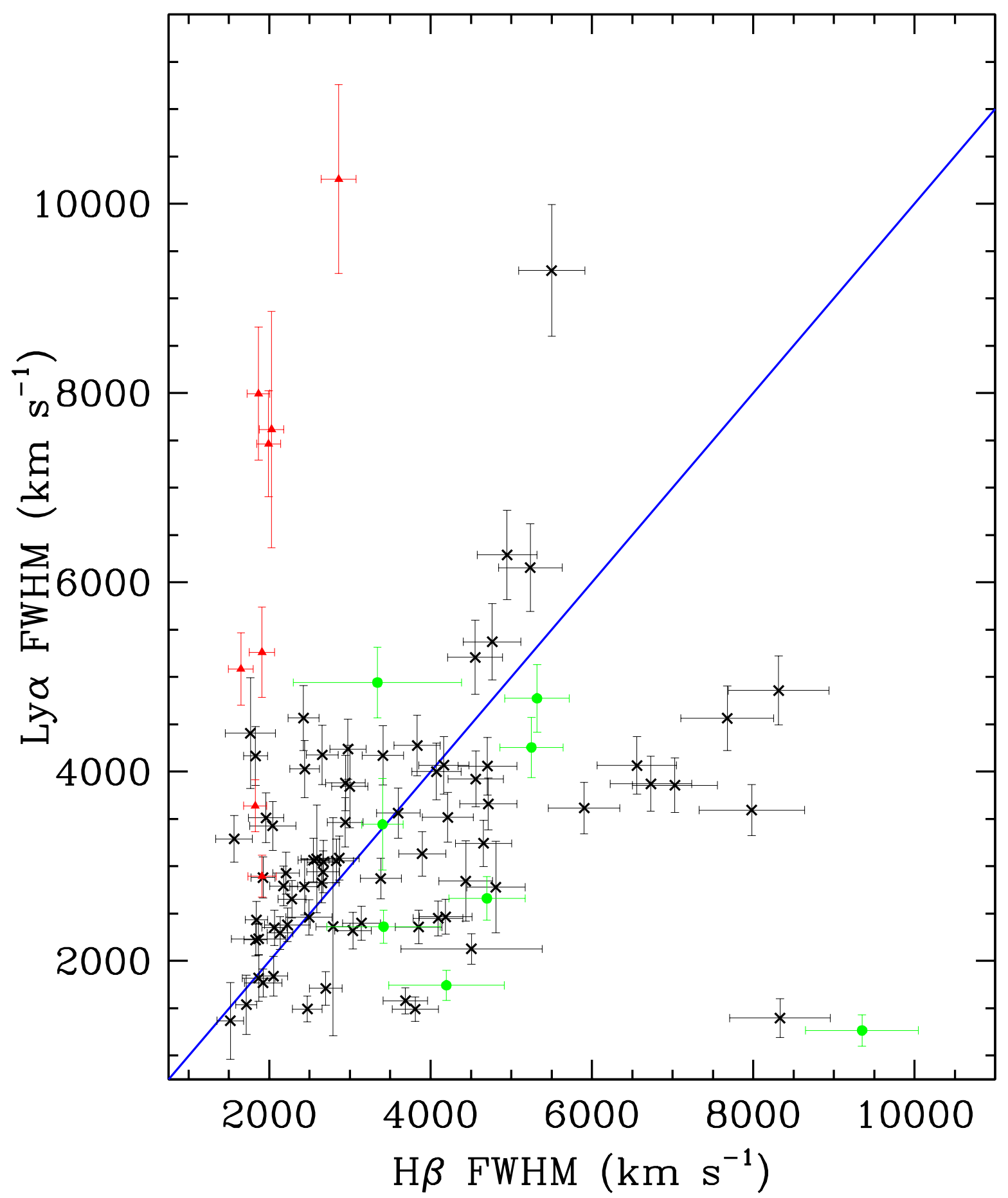

Figure 8. FWHMs of Ly $\alpha$ vs. H $\beta$. The blue line marks an ideal situation of equal FWHMs. The Pearson coefficient of 0.08 suggests a poor correlation. The distributions of Fe II-strong (red) and Fe II-weak (green) quasars are noticeably different. 


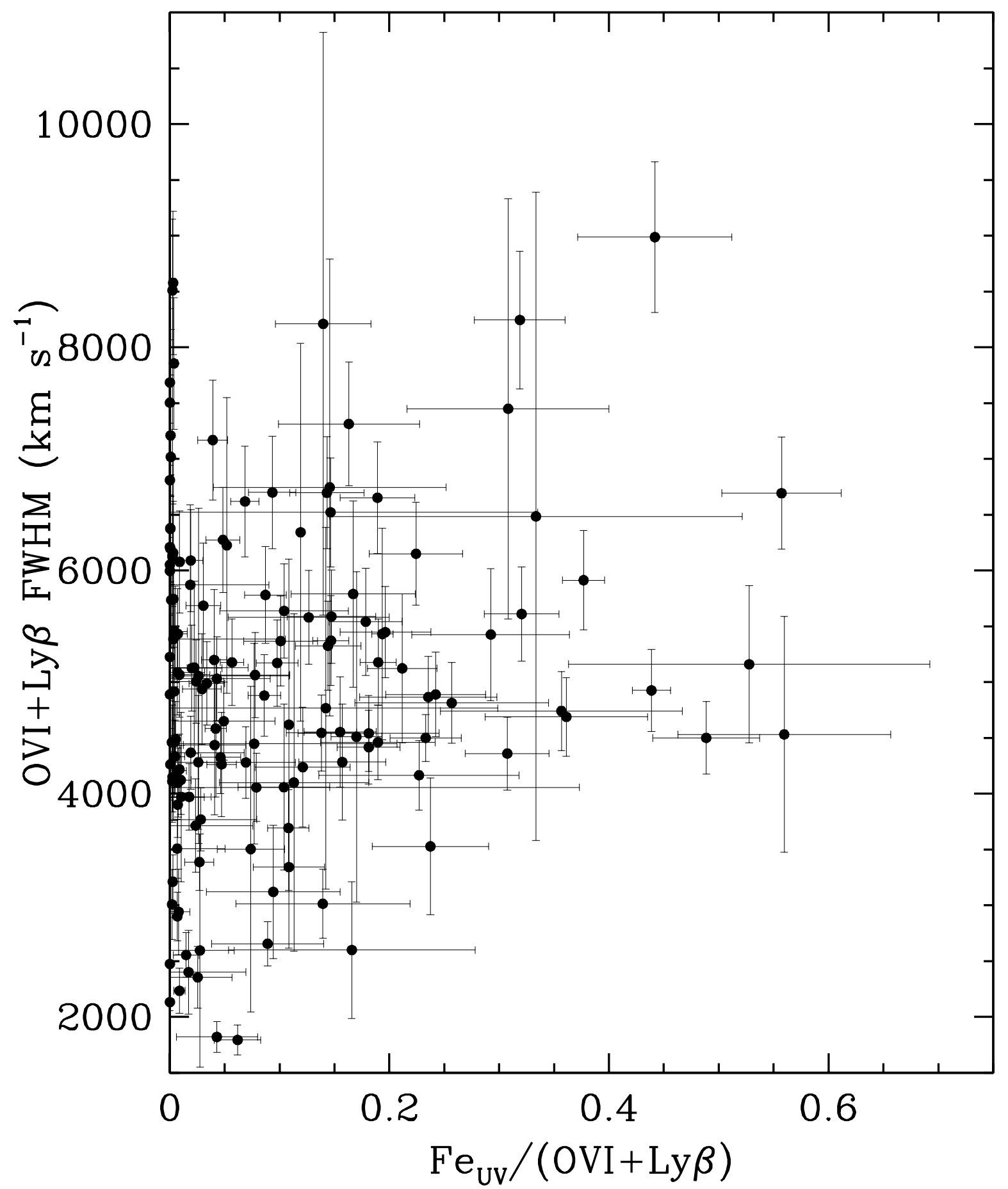

Figure 9. Ratios of FeUv/(Ly $\beta+\mathrm{O}$ vi) vs. line widths of Ly $\beta+\mathrm{O}$ vi. Quasars with strong Fe II features tend to display broader emission, contrary to a trend in the optical band. 
Table 1. Emission Line Strengths in Fe II-Strong Quasars

\begin{tabular}{|c|c|c|c|c|c|c|c|c|c|c|}
\hline \multicolumn{2}{|l|}{ Quasar } & \multicolumn{6}{|c|}{ Equivalent Width $(\AA)$} & \multicolumn{2}{|c|}{ Flux Ratio } & \multirow[t]{2}{*}{ S06 } \\
\hline Name & Redshift & $\begin{array}{c}\mathrm{Fe} \text { II } \\
\lambda 4565\end{array}$ & $\begin{array}{c}\mathrm{H} \beta \\
\lambda 4861\end{array}$ & $\begin{array}{l}\mathrm{Fe}_{\mathrm{UV}} \\
\lambda 1123\end{array}$ & $\begin{array}{c}\mathrm{Ly} \beta+\mathrm{O} \text { vI } \\
\lambda \lambda 1026,1034\end{array}$ & $\begin{array}{c}\text { Ly } \alpha \\
\lambda 1216\end{array}$ & $\begin{array}{c}\mathrm{C} \text { II } \\
\lambda 1335\end{array}$ & $\mathrm{Fe} \mathrm{II}_{\mathrm{opt}} / \mathrm{H} \beta$ & $\mathrm{Fe}_{\mathrm{UV}} /(\mathrm{Ly} \beta+\mathrm{O} \mathrm{VI})$ & \\
\hline SDSS1010+3003 & 0.2558 & $68.8 \pm 0.7$ & $48.5 \pm 0.1$ & $5.0 \pm 0.1$ & $10.2 \pm 0.1$ & $53 \pm 3$ & $2.1 \pm 0.5$ & $1.42 \pm 0.06$ & $0.49 \pm 0.05$ & $\mathrm{NO}$ \\
\hline SDSS0904+4007 & 0.4100 & $78.9 \pm 2.1$ & $61.4 \pm 0.1$ & $6.0 \pm 0.1$ & $10.8 \pm 0.1$ & $95 \pm 4$ & - & $1.28 \pm 0.07$ & $0.56 \pm 0.05$ & $\mathrm{NO}$ \\
\hline SDSS1619+2543 & 0.2685 & $81.2 \pm 0.5$ & $64.2 \pm 0.1$ & $5.7 \pm 0.1$ & $10.2 \pm 0.2$ & $87 \pm 2$ & $3.2 \pm 0.8$ & $1.26 \pm 0.02$ & $0.56 \pm 0.10$ & $\mathrm{NO}$ \\
\hline SDSS1202-0129 & 0.1504 & $50.6 \pm 1.4$ & $40.1 \pm 0.1$ & $7.4 \pm 0.6$ & $22.1 \pm 0.1$ & $42 \pm 2$ & - & $1.26 \pm 0.09$ & $0.33 \pm 0.19$ & $\mathrm{NO}$ \\
\hline SDSS1301+5902 & 0.4764 & $74.9 \pm 0.5$ & $59.4 \pm 0.1$ & $3.5 \pm 0.2$ & $7.9 \pm 0.1$ & $56 \pm 5$ & - & $1.26 \pm 0.02$ & $0.44 \pm 0.07$ & YES \\
\hline SDSS0102-0039 & 0.2943 & $90.5 \pm 0.7$ & $74.8 \pm 0.1$ & $5.0 \pm 0.1$ & $15.8 \pm 0.1$ & $61 \pm 5$ & $2.6 \pm 3.0$ & $1.21 \pm 0.02$ & $0.32 \pm 0.04$ & $\mathrm{NO}$ \\
\hline SDSS0912+2957 & 0.3054 & $50.2 \pm 1.7$ & $41.5 \pm 0.1$ & $2.9 \pm 0.1$ & $13.9 \pm 0.1$ & $87 \pm 2$ & $13.9 \pm 1.2$ & $1.21 \pm 0.08$ & $0.21 \pm 0.03$ & $\mathrm{NO}$ \\
\hline SDSS1233-0031 & 0.4712 & $64.8 \pm 2.1$ & $55.2 \pm 0.1$ & $1.9 \pm 0.2$ & $6.1 \pm 0.2$ & - & - & $1.17 \pm 0.09$ & $0.31 \pm 0.09$ & $\mathrm{NO}$ \\
\hline SDSS1323+3430 & 0.4429 & $67.0 \pm 7.3$ & $75.6 \pm 0.1$ & $5.1 \pm 0.3$ & $9.7 \pm 0.1$ & $86 \pm 5$ & - & $0.89 \pm 0.11$ & $0.53 \pm 0.16$ & $\mathrm{NO}$ \\
\hline SDSS1519+2838 & 0.2690 & $55.2 \pm 2.2$ & $62.9 \pm 0.1$ & $12.4 \pm 0.1$ & $28.3 \pm 0.1$ & - & - & $0.88 \pm 0.04$ & $0.44 \pm 0.02$ & $\mathrm{NO}$ \\
\hline Average /Deviation & $0.33 \pm 0.10$ & $68.2 \pm 12.8$ & $58 \pm 12$ & $5.5 \pm 2.8$ & $13 \pm 7$ & $71 \pm 19$ & $5.5 \pm 4.9$ & $1.19 \pm 0.16$ & $0.42 \pm 0.11$ & \\
\hline
\end{tabular}

Table 2. Emission Line Widths in Fe II-Strong Quasars

\begin{tabular}{cccc}
\hline Quasar & \multicolumn{3}{c}{ FWHM $\left(\mathrm{km} \mathrm{s}^{-1}\right)$} \\
\cline { 2 - 4 } & Ly $\beta+\mathrm{O}$ vI & Ly $\alpha$ & $\mathrm{H} \beta$ \\
& $\lambda \lambda 1026,1034$ & $\lambda 1216$ & $\lambda 4861$ \\
\hline SDSS1010+3003 & $4500 \pm 325$ & $5262 \pm 476$ & $1909 \pm 157$ \\
SDSS0904+4007 & $6693 \pm 502$ & $7616 \pm 1249$ & $2028 \pm 152$ \\
SDSS1619+2543 & $4531 \pm 1056$ & $3639 \pm 273$ & $1826 \pm 143$ \\
SDSS1202-0129 & $6485 \pm 2906$ & $7463 \pm 560$ & $1992 \pm 149$ \\
SDSS1301+5902 & $8987 \pm 674$ & $10260 \pm 997$ & $2860 \pm 215$ \\
SDSS0102-0039 & $8244 \pm 618$ & $7993 \pm 703$ & $1866 \pm 140$ \\
SDSS0912+2957 & $5123 \pm 668$ & $2895 \pm 220$ & $1911 \pm 176$ \\
SDSS1233-0031 & $7449 \pm 1882$ & - & $3678 \pm 277$ \\
SDSS1323+3430 & $5160 \pm 704$ & $5085 \pm 381$ & $1649 \pm 154$ \\
SDSS1519+2838 & $4925 \pm 369$ & - & $2074 \pm 156$ \\
\hline Average /Deviation & $6210 \pm 1529$ & $6277 \pm 2315$ & $2179 \pm 585$ \\
\hline
\end{tabular}


Table 3. Emission Line Strengths in Fe II-Weak Quasars

\begin{tabular}{|c|c|c|c|c|c|c|c|c|c|c|}
\hline \multicolumn{2}{|l|}{ Quasar } & \multicolumn{6}{|c|}{ Equivalent Width $(\AA)$} & \multicolumn{2}{|c|}{ Flux Ratio } & \multirow[t]{2}{*}{ S06 } \\
\hline Name & Redshift & $\begin{array}{c}\text { Fe II } \\
\lambda 4565\end{array}$ & $\begin{array}{c}\mathrm{H} \beta \\
\lambda 4861\end{array}$ & $\begin{array}{l}\mathrm{Fe}_{\mathrm{UV}} \\
\lambda 1123\end{array}$ & $\begin{array}{c}\mathrm{Ly} \beta+\mathrm{O} \text { VI } \\
\lambda \lambda 1026,1034\end{array}$ & $\begin{array}{c}\text { Ly } \alpha \\
\lambda 1216\end{array}$ & $\begin{array}{c}\mathrm{C} \text { II } \\
\lambda 1335\end{array}$ & $\mathrm{Fe} \mathrm{II}_{\mathrm{opt}} / \mathrm{H} \beta$ & $\mathrm{Fe}_{\mathrm{UV}} /(\mathrm{Ly} \beta+\mathrm{O}$ VI $)$ & \\
\hline SDSS1010+2559 & 0.5115 & $4.8 \pm 2.1$ & $76.8 \pm 0.1$ & $0.1 \pm 0.1$ & $24.0 \pm 0.1$ & - & - & $0.06 \pm 0.03$ & $0.00 \pm 0.01$ & $\mathrm{NO}$ \\
\hline SDSS1107+1628 & 0.6302 & $0.0 \pm 0.1$ & $98.2 \pm 0.1$ & $0.0 \pm 1.2$ & $16.7 \pm 0.1$ & $93 \pm 8$ & $0.0 \pm 0.1$ & $0.00 \pm 0.01$ & $0.00 \pm 0.01$ & YES \\
\hline SDSS1617+0638 & 0.2293 & $0.0 \pm 0.1$ & $102.5 \pm 0.1$ & $0.9 \pm 0.6$ & $98.7 \pm 0.1$ & $512 \pm 6$ & $0.1 \pm 1.1$ & $0.00 \pm 0.01$ & $0.01 \pm 0.01$ & $\mathrm{NO}$ \\
\hline SDSS1330+3119 & 0.2422 & $37.1 \pm 3.7$ & $173.3 \pm 0.1$ & $0.8 \pm 0.5$ & $31.1 \pm 0.1$ & $106 \pm 2$ & $0.4 \pm 0.5$ & $0.21 \pm 0.02$ & $0.03 \pm 0.01$ & $\mathrm{NO}$ \\
\hline SDSS1210+3157 & 0.3891 & $0.5 \pm 0.7$ & $81.7 \pm 0.1$ & $0.9 \pm 1.1$ & $20.2 \pm 0.1$ & $173 \pm 4$ & - & $0.01 \pm 0.01$ & $0.04 \pm 0.05$ & $\mathrm{NO}$ \\
\hline SDSS1620+1736 & 0.5551 & $0.4 \pm 3.0$ & $99.8 \pm 0.1$ & $0.0 \pm 29.7$ & $8.7 \pm 0.2$ & $96 \pm 6$ & $0.1 \pm 0.1$ & $0.00 \pm 0.03$ & $0.00 \pm 0.10$ & YES \\
\hline SDSS1220+0203 & 0.2404 & $0.5 \pm 1.5$ & $72.7 \pm 0.1$ & $0.2 \pm 2.0$ & $20.4 \pm 0.1$ & $99 \pm 5$ & - & $0.01 \pm 0.02$ & $0.01 \pm 0.02$ & $\mathrm{NO}$ \\
\hline SDSS1528+2825 & 0.4473 & $8.3 \pm 3.3$ & $77.1 \pm 0.1$ & $0.0 \pm 0.1$ & $21.8 \pm 0.1$ & $133 \pm 19$ & - & $0.11 \pm 0.04$ & $0.00 \pm 0.01$ & $\mathrm{NO}$ \\
\hline SDSS1120+4235 & 0.2265 & $11.3 \pm 1.9$ & $110.7 \pm 0.1$ & $0.0 \pm 0.1$ & $20.0 \pm 0.1$ & - & - & $0.10 \pm 0.02$ & $0.00 \pm 0.01$ & $\mathrm{NO}$ \\
\hline SDSS0928+6025 & 0.2954 & $19.2 \pm 1.3$ & $50.6 \pm 0.1$ & $1.2 \pm 0.6$ & $12.8 \pm 0.1$ & $90 \pm 29$ & $2.1 \pm 0.8$ & $0.38 \pm 0.03$ & $0.09 \pm 0.06$ & $\mathrm{NO}$ \\
\hline Average /Deviation & $38 \pm 0.14$ & $8.2 \pm 11.4$ & $94 \pm 31$ & $0.4 \pm 0.4$ & $27 \pm 24$ & $163 \pm 135$ & $0.6 \pm 0.8$ & $0.09 \pm 0.12$ & $0.02 \pm 0.03$ & \\
\hline
\end{tabular}

Table 4. Emission Line Widths in Fe II-Weak Quasars

\begin{tabular}{cccc}
\hline Quasar & \multicolumn{3}{c}{ FWHM $\left(\mathrm{km} \mathrm{s}^{-1}\right)$} \\
\cline { 2 - 4 } & Ly $\beta+\mathrm{O}$ vI & Ly $\alpha$ & $\mathrm{H} \beta$ \\
& $\lambda \lambda 1026,1034$ & $\lambda 1216$ & $\lambda 4681$ \\
\hline SDSS1010+2559 & $4916 \pm 859$ & - & $2411 \pm 181$ \\
SDSS1107+1628 & $8510 \pm 638$ & $4774 \pm 358$ & $5320 \pm 399$ \\
SDSS1617+0638 & $2234 \pm 202$ & $1742 \pm 157$ & $4196 \pm 717$ \\
SDSS1330+3119 & $3387 \pm 254$ & $1266 \pm 165$ & $9349 \pm 701$ \\
SDSS1210+3157 & $5030 \pm 377$ & $4256 \pm 319$ & $5251 \pm 394$ \\
SDSS1620+1736 & $5385 \pm 1624$ & $4942 \pm 371$ & $3341 \pm 1045$ \\
SDSS1220+0203 & $3970 \pm 760$ & $2362 \pm 177$ & $3417 \pm 701$ \\
SDSS1528+2825 & $4890 \pm 367$ & $3443 \pm 484$ & $3403 \pm 255$ \\
SDSS1120+4235 & $2133 \pm 796$ & - & $4919 \pm 369$ \\
SDSS0928+6025 & $3120 \pm 596$ & $2661 \pm 232$ & $4698 \pm 473$ \\
\hline Average /Deviation & $4358 \pm 1773$ & $3181 \pm 1301$ & $4630 \pm 1816$ \\
\hline \hline
\end{tabular}

This paper has been typeset from a $\mathrm{TEX}_{\mathrm{E}} / \mathrm{LAT} \mathrm{EX}$ file prepared by the author. 